\title{
Usefulness of a Partograph to improve outcomes: Scientific Evidence
}

\author{
Weerasekara $\mathrm{D}^{1}$
}

\section{INTRODUCTION}

The partograph was first introduced in 1954 by Friedman graphically depicting the dilatation of the cervix during labour ${ }^{1}$. Philpott and Castle in 1972 developed Friedman's concept into a tool for monitoring labour by adding the action and alert lines ${ }^{2}$. The current patograph includes different variables such as fetal heart rate, dilatation of the cervix, contractions, and maternal pulse and blood pressure plotted on a preprinted paper. Therefore the current partograph is designed to monitor not only the progress of labour but also the condition of the mother and fetus during labour.

The partogram provides a graphical record of the progress of labour and it is considered to be a valuable tool in the management of women in labour. The usefulness of a partogram are that;

1. It depicts the progress of labour at a glance

2. It enables failure to progress to be readily recognized

3. It is simple to use

4. Provides a practical teaching aid

5. Is an efficient means of exchange of technical information about labour progress between teams of caregivers $^{3}$

Partographs often contain alert and action lines. An alert line represents slowest $10 \%$ of primigravid women's labour progress. An action line is placed a number of hours after the alert line (usually two to four hours) to

\footnotetext{
1 Chair professor of Obstetrics and Gynaecology, South Asian Institute of Technology and Medicine (SAITM)
}

Correspondence: Prof. Deepal Weerasekara

E-mail: deepalsenaka@yahoo.co.uk prompt effective management of slow progress of labour ${ }^{4}$. It is important to detect prolonged labour as this can lead to post partum haemarrhage and infections ${ }^{5}$. These issues are even more important in low resource settings due to non-availability of aseptic procedures in vaginal examinations. In a setting where a difficult instrumental delivery or an emergency caesarean section cannot be carried out arrangements can be made to transfer the woman to a hospital where these facilities are available if the progress crosses the alert line.

\section{SCIENTIFIC EVIDENCE ON USEFULNESS OFA PARTOGRAPH}

In 1994 the World Health Organization (WHO 1994) conducted a prospective non-randomised study of 35,484 women in South East Asia and concluded that the partograph was a necessary tool in the management of labour and recommended its universal application ${ }^{6}$. This study was performed in four pairs of hospitals and a staged approached was adopted. Introduction of the partogram, and agreed management protocol, reduced prolonged labour from $6.4 \%$ to $3.4 \%$, the proportion of labour requiring augmentation from $9.9 \%$ to $8.3 \%$, and still births from $0.5 \%$ to $0.3 \%$.

There is also evidence to suggest that midwives find the partogram to have practical benefits in terms of ease of use, time resourcefulness, continuity of care and educational assistance . $^{7}$

Partographs were initially introduced in rural settings with limited medical input and resources. Argument that partograph use is not affected by racial, cultural and socioeconomic differences, led to the approach of introducing partographs to both high income and middle income countries. As a consequence some have questioned its effectiveness when used in high-income countries $^{8}$. Therefore transferability of such a tool for clinical practice needs consideration. Maintaining partographs may restrict clinical practice, reduce midwife autonomy and limit the flexibility to treat each woman as an individual. Also there is a concern that partographs can create unnecessary interference ${ }^{9}$. All women in labour will not progress at the same rate. By assuming this, partograph use can have adverse effects such as increased rates of artificial rupture of membranes, oxytocin augmentation and use of analgesia resulting in a more negative labour experience.

In two randomized trials comparing 1590 participating women pooled data show that there was no significant difference between groups in caesarean section (Risk ratio (RR) 0.64, $95 \%$ confidence interval CI 0.24 to 1.7 ), instrumental vaginal delivery (RR $1.00,95 \%$ CI 0.85 to 1.17), or Apgar score less than 7 at five minutes (RR $0.77,95 \%$ CI 0.29 to 2.06$)^{10,11}$. Two studies showed different results for caesarean sections. The study done in the low resource settings showed that the caesarean section rate was lower in the partogram group ${ }^{10}$. In the high resource setting there was no difference in the two groups ${ }^{11}$. In another data analysis involving six studies partogram versus no partogram showed no evidence of significant difference between partogram and no partogram in caesarean section rates ${ }^{12}$.

Two different randomized trials with partograms of two-hour action line and partogram with four hour action line did not show any significant difference in the caesarean section rates (RR 1.06, 95\% CI 0.85 to 1.32 , $\mathrm{n}=3601)^{13,14}$. A randomized trial comparing a partogram with an alert line only with a partogram with an alert and action line showed that caesarean section rate was lower in the alert line only group (RR $0.68,95 \%$ CI 0.50 to $0.93, n=694)^{15}$. There was 
no difference in any of the remaining maternal or neonatal outcomes.

\section{DISCUSSION}

Current evidence regarding the use of partograph is controversial.

At present evidence from studies comparing partograph with no partograph shows no difference in caesarean section rates, duration of labour, oxytocin augmentation, amniotomy, epidural use, and use of antibiotics in labour, Apgar scores or admissions to neonatal intensive care unit. These findings were based on two randomized trials and the findings may have been influenced by the relatively high percentage of non-compliance in completing the partograph $(20 \%)$ or the cross contamination of the care by staff or both $^{10,11}$. Based on the evidence of these two trials it cannot be recommended that maintaing partograph is mandatory in labour. However many units in high and low income settings use partographs and have reported benefits in terms of ease of recording, provision of pictorial overview of progress, training of clinicians and transferring of care $^{16}$. Therefore until more definite evidence is available the use of partograph should be determined by clinical and maternal preference. Also findings of these two studies cannot be extrapolated to units where the partograph is currently in use. In low-resource settings prolonged labour and delay in decision making are important causes of adverse obstetric outcomes. Owing to resource constraints in such settings, it is usually not possible to monitor each woman continuously throughout the duration of labour. In these situations partograph serves as a simple and inexpensive tool to monitor labour in a cost-effective way. One-case control study from Pakistan found the partogram to reduce the frequency of prolonged labour, post-partum haemorrhage, ruptured uterus, puerperal sepsis, perinatal and maternal morbidity and mortality ${ }^{18}$. Removing the partograph as opposed to introducing it may produce different findings. Therefore as at present it is not advisable to recommend any change in the current routine use of of partograph or use of a specific type of partograph.

Data from trials comparing the different placement of action lines show little difference in instrumental vaginal delivery, maternal morbidity, performance of artificial rupture of membranes, blood loss less than 500 mls, epidural use, and number of vaginal examinations ${ }^{13,14}$. When the two hour action line was compared with the four hour action line the only difference found was an increase in oxytocin augmentation in the two hour arm (RR 1.14, 95\% CI 1.05 to 1.22 , $\mathrm{n}=3601)$. There were no differences in any neonatal outcomes such as cord $\mathrm{pH}$ less than 7.1, Apgar scores less than seven at five minutes, and admission to special care baby unit, serious morbidity or perinatal death.

Trial comparing a partograph with an alert line with one with an alert and action line clearly demonstrated a difference in caesarean section rates with the only alert line partograph having the lower rate (RR $0.68,95 \% \mathrm{CI}$ 0.50 to $0.93, n=694)^{15}$.

Given the limited number of trials on partograms and the heterogeneity, it is difficult to offer any recommendations for the routine use of specific types of partogram ${ }^{17}$. None of the analysed studies were multi-centred and all study units had different labour ward guildlines. Interestingly both studies from low income countries showed a statistically significant difference in caesarean section rates ${ }^{14,10}$. A large multicenter randomized trial using a uniform labour ward protocol is necessary to determine the usefulness of a partograph to improve outcomes. This is especially needed in low-resource settings. Factors such as parity, previous obstetrical outcome, and women's satisfaction and obstetricians or birth attendant's experience with the use of the partograph should also needs to be analyzed by stratification of participants ${ }^{19}$.

\section{REFERENCES}

1. Friedman E, Graphic analysis of labour. American journal of obstetrics and Gynecology, 1954; 68:1568-75.

2. Philpott $\mathrm{RH}$, Castle WM, Cervicographs in the management of labour in primigravidae. Journal of Obstetrics and Gynaecology of the British Commonwealth 1972;79:592-598.

3. EnkinM, Keirse NC, Neilsen J, et al. Monitoring the progress of labour. A guide to effective care in pregnancy and childbirth 3rd edition, Oxford university press 2000,p 281-8

4. Lavender T, Hart A, Symth R, Effect of partogram use on outcomes for women in spontaneous labour at term. The Cochrane Collaboration. Published by John Wiley \& Sons, Ltd. 2009, 1-22.

5. Neilson J, Lavender T, Quenby S,Wray S. Obstructed labour. British Medical Bulletin, 2003; 67:191-204.

6. World Health Organization. World Health Organization partograph in management of labour. Lancet 1994: 343: 1399-404.

7. Lavender $\mathrm{T}$, Malcolmson $\mathrm{L}$. Is the partogram a help or a hindrance? Practising Midwife 1999: 2: 23-7.

8. Groeschel N, Glover P. The partograph. Used daily but rarely questioned. Australian Journal of Midwifery 2001: 14: 22-7.

9. Walraven GE. WHO partograph. Lancet 1994: 344: 617.

10. Walss-Rodriguez RJ, Gudino-Ruiz F, Tapia-Rodrguez S. Comparative study between Friedman's partogram and conventional descriptive partogram. Ginecologia y Obstetricia de Mexico 1987:55:318-22.

11. Windrim R, Seaward G, Hodnett E, Akoury H, Kingdom J, Salenieks ME, et al. A randomized controlled trial of a bedside partogram in the active management of primiparous labour. Journal of Obstetrics and Gynaecology Canda: JOGC 2006: 29(1): 27-34.

12. Leanza V, Leanza G, Monte S. A didactic protocol for labour and delivery: the partogram. Minerva ginecologica 08/2011:63(4):352-32.

13. Lavender $T$, Walkinshaw $S$, Alfirevic $Z$. Partogram action line study: a randomized trial. British Journal of Obstetrics and Gynaecology 1998:105:976-80. 
14. Lavender T, Alfirevic Z, Walkinshaw $S$. Effect of different partogramaction lines on birth outcomes. Obstetrics \& Gynaecology 2006:108:295-302.

15. Pattinson RC, Howarth GR, Mdluli W, Macdonald AP, Makin JD, Funk M. Aggressive or expectant management of labour: a randomized trial. BJOG: an international journal of obstetrics and gynaecology 2003, 110:457-61.
16. Lavender $T$, Lugina $H$, Sumith $H$, The partograph: A life saving toolfor African Midwives. Tropical Doctor 2007: 37 (3): 191-192.

17. Lavender T, Hart A, Smyth RMD. Effect of partogram use on outcomes for women in spontaneous labour at term (Review) 2009 The Cochrane Collaboration. Published by John Wiley \& Sons, Ltd.
18. Javed I, Bhutta S, Shoaib T, Role of partogram in preventing prolonged labour. Journal of the Pakistan Medical Association 2007;57(8):408-411.

19. Soni BL. Effect of partogram use on outcomes for women in spontaneous labour at term: RHL commentary (revised: 1 June 2009) The WHO Reproductive Health Library; Geneva: World Health Organization. 